\title{
Prevalence and risk factors of mental disorders during the COVID- 19 pandemic in Bangladesh: a systematic review
}

Ismail Hosen

Jahangirnagar University

Firoj al Mamun

Jahangirnagar University

Mohammed A. Mamun (D mamunphi46@gmail.com)

CHINTA Research Bangladesh https://orcid.org/0000-0002-1728-8966

\section{Research Article}

Keywords: COVID-19 and Psychological Impact, Mental Health in Bangladesh, Depression, Anxiety, Stress, PTSD, Systematic Review, Prevalence and Risk Factors, Bangladesh

Posted Date: June 28th, 2021

DOI: https://doi.org/10.21203/rs.3.rs-631435/v1

License: @ (i) This work is licensed under a Creative Commons Attribution 4.0 International License. Read Full License 


\section{Abstract \\ Background}

The newly incepted Coronavirus Disease 2019 (COVID-19) has become a global public health threat, subsequently impacting individuals' psychological wellbeing along with the risk of physical complications. Under the circumstance, it is essential to synthesize the existing evidence on psychological consequences regarding potential implications for policymaking and practice. Thus, a systematic attempt was compiled to review the Bangladeshi literature related to common mental health problems (i.e., depression, anxiety and stress) conducted during the COVID-19 pandemic.

\section{Methods}

Adhering to the PRISMA guideline, a systematic literature search was performed using Scopus, Medline or PubMed, Google Scholar, ResearchGate and Google, between 20 December 2020 and 5 March 2021, followed by predetermined eligibility criteria. The inclusion criteria for this review were observational studies concerning at least one common mental disorder (i.e., stress, depression and anxiety) prevalence and risk factors reported after the inception of the COVID-19 pandemic in Bangladesh and being published in peer-reviewed journals or preprint servers in the English language. A total of 24 preprint and peer-reviewed articles were finally recruited after meeting the inclusion criteria.

\section{Results}

Out of 24 studies, utmost of the investigation was directed among the general population $(n=9)$, followed by diverse cohorts such as students $(n=7)$, healthcare professionals $(n=4)$, COVID-19 infected individuals $(n=1)$, quarantined individuals $(n=1)$, wage-earners $(n=$ $1)$, and bankers $(n=1)$. However, the prevalence range of anxiety $(10.6-81.8 \%)$, depression $(12.1-82.4 \%)$, and stress $(11.1-85.6 \%)$ were found among altogether available literature during the COVID-19 pandemic in Bangladesh. Moreover, the associated risk factors of mental disorders included gender, age, residence area, family size, monthly family income, educational status, marital status, physical exercise, smoking, alcohol use, personal protective equipment, fear of COVID-19, presence of chronic illness, being unemployed and exposure of COVID-19 related news and social media.

\section{Conclusions}

This systematic review provides baseline data on mental disorders across various Bangladeshi cohorts, which are anticipated to be helpful to the respective authorities of the country implement cohort-specific mental health strategies.

\section{Introduction}

As stated by the United Nations Development Program (UNDP) (1), the newly incepted Coronavirus Disease 2019 (COVID-19) pandemic has become the most challenging global issue since World War II. This contagious disease has already spread out within all countries and territories worldwide after its first appearance in late December 2019 at Wuhan, China, caused by a newly discovered virus named severe acute respiratory syndrome coronavirus 2 (SARS-CoV-2) (2). Regardless of the unpredictable and uncontrolled situation of the viral infection, the World Health Organization (WHO) declared this outbreak as a pandemic on 11 March $2020(2,3)$. The virus is rapidly transmittable; after that, approximately $124 \mathrm{M}$ confirmed COVID-19 cases, including $2.74 \mathrm{M}$ deaths, are reported globally as of $23 \mathrm{March}$ 2021, whereas 577K and 8,720 respectively are the statistics for Bangladesh.

People infected with the SARS-CoV-2 manifests both symptomatic and asymptomatic signs and symptoms, including fever, cough, fatigue, headache, myalgia, muscle pain, breath shortness, acute upper respiratory tract distress, diarrhea etc. $(4,5)$, which are the typical features of mental health problems (6-8). The higher rates of morbidity and mortality impact individuals' quality of life, leading to individuals worsening psychological state, although not being infected with the SARS-CoV-2. However, to alleviate this contagious outbreak at the community level, some of the non-therapeutic public health interventions and measures were already implemented globally, including countrywide lockdown, home or institution-based isolation or quarantine, shutting down all kinds of public activities along with closing educational institutions, especially restriction in social and community movements etc. $(1,9,10)$. Prior studies 
observed that people under these measures reported substantial mental health impacts, which led to people disliking the importance of social restriction measures suppressing the viral infection (11).

In Bangladesh, the first COVID-19 case was identified on 8 March 2020 (12). After some of that point, Bangladesh's authority seized countrywide lockdown with the title of 'general holidays' to mitigate the outbreak at the community level, which was a significant challenge for a lower- and middle-income country with limited resources in the healthcare settings (12). Under these circumstances, many people became cut down from their jobs and unemployed without the hope of alternative new jobs $(1,13)$. Consequently, the jobless situation led people to suffer from higher rates of mental instabilities (14). Considering the mental problems related to unemployment and economic crisis, self-harm and suicidal incidences are also observed in Bangladesh. For instance, a study reported all but one of the COVID-19 related suicide cases occurred as of economic and financial problems within the first three weeks of April 2020 [14]. In addition, the uncertainty about going back to everyday life, fear of being infected, social and economic disruption, rising social negligence, difficulties in getting proper treatment as of the overwhelming number of patients etc. generally increase psychological suffering, including post-traumatic stress disorder (PTSD), insomnia, phobia, fear, anxiety, depression etc. (15-17).

In Bangladesh, after the first COVID-19 suicide incidence on 25 March 2020, epidemiological studies concerning mental health problems to identify risky individuals were recommended [19], and many studies have already been conducted across the country. But, there is no beyond a systematic evaluation to better understand the impact of the COVID-19 pandemic on people's psychological health. Therefore, this study attempts to review the Bangladeshi studies concerning the COVID-19 pandemic-related mental health problems such as depression, anxiety and stress (more specifically, studies reporting the prevalence and/or risk factors of these mental health outcomes); which in anticipated facilitating appropriate mental health strategies.

\section{Methods}

Search Strategies

For conducting a systematic review, Preferred Reporting Items for Systematic Reviews and Meta-Analyses (PRISMA) guideline (18) was adhered to the present study. Literature searches were conducted between 20 December 2020 and 5 March 2021, using a group of bounded keywords in the relevant databases. The searching keywords included three types of items, (i) exposure (e.g., 'COVID-19', 'Coronavirus', SARS-Cov-2, 'COVID-19 pandemic'), (ii) outcome of interest (e.g., 'mental health', 'mental disorder', 'psychological impact', 'depressive disorders', 'depression', 'anxiety disorder', 'anxiety', 'stress', 'stress disorders'), and (iii) country (e.g., 'Bangladesh'). The searches were conducted with the combination of these three types of keywords.

Data Eligibility Criteria

For being included in the present review, every article must fulfil the following requirement of - (i) being a Bangladeshi study, (ii) being conducted after the inception of the COVID-19 pandemic, (iii) being an observational study (cross-sectional or longitudinal) in nature, (iv) being concerned with at least one common mental disorders (i.e., PTSD or stress, depression and anxiety), (v) reporting the prevalence and/or risk factors of these disorders, (vi) being published in peer-reviewed journals or preprint servers, (vii) being published in the English language.

Data Collection Procedure

First of all, a comprehensive literature search was conducted using the databases of Medline or PubMed to identify articles related to the prevalence and/or risk factors of depression, anxiety and stress reported during the COVID-19 pandemic in Bangladesh. Later on, Scopus, PsycINFO, Web of Science, CINAHL, Google Scholar, ResearchGate, and even Google search engine was used to retrieve missing peerreviewed and preprint articles. Along with that, additional missing articles were tried obtaining from the bibliographic references of the retrieved articles. Therefore, after a preliminary search conducted by the principal investigator, other investigators subsequently re-circled the procedure to ensure articles were not excluded from this review. A total of 175 articles were identified, whereas the 'titles and abstracts' of the retrieved articles were scanned to adhere to the study inclusion criteria of the present review. To the end, a total of 24 articles were included in this review after confirming all of the requirements (Fig. 1).

\section{Data Extraction}

In Microsoft Excel, a data extraction file was designed to make necessary changes from the recruited articles. After that, data were demonstrated by following records: information about the author and publication year, study design, sampling and data collection 
method, specific group and sample size, time for data collection, assessments tool along with cutoff value and the prevalence and risk factors of PTSD, stress, depression and anxiety by the principal investigator. After the independent investigation and cross-checking by all the investigators, the disagreements were solved in the final file as presented in Table 1. 
Table 1

Characteristics of the included literatures to the present review

\begin{tabular}{|c|c|c|c|c|c|c|c|c|}
\hline $\begin{array}{l}\text { Authors } \\
\text { and } \\
\text { publication } \\
\text { year }\end{array}$ & $\begin{array}{l}\text { Study } \\
\text { design and } \\
\text { Sampling } \\
\text { procedure }\end{array}$ & $\begin{array}{l}\text { Data } \\
\text { collection } \\
\text { method } \\
\text { and time }\end{array}$ & $\begin{array}{l}\text { Sample } \\
\text { size }\end{array}$ & $\begin{array}{l}\text { Specific } \\
\text { group and } \\
\text { mean age }\end{array}$ & $\begin{array}{l}\text { Assessment } \\
\text { scale with } \\
\text { cutoff score }\end{array}$ & $\begin{array}{l}\text { Prevalence } \\
\text { rate(s) }\end{array}$ & Risk factors & $\begin{array}{l}\text { Assessment } \\
\text { criteria }\end{array}$ \\
\hline $\begin{array}{l}\text { Sayeed et } \\
\text { al. }(2020) \\
(19)\end{array}$ & $\begin{array}{l}\text { Cross- } \\
\text { sectional; } \\
\text { Snowball }\end{array}$ & $\begin{array}{l}\text { Web } \\
\text { survey; } \\
\text { April 29- } \\
\text { May 7, } \\
2020\end{array}$ & 589 & $\begin{array}{l}\text { Students, } \\
\text { Age: NR }\end{array}$ & $\begin{array}{l}\text { DASS-21; } \\
\text { Cutoff: } \\
\text { anxiety } \geq 7 \text {, } \\
\text { depression } \\
\geq 10 \text {, stress } \\
\geq 11 \text { ) }\end{array}$ & $\begin{array}{l}\text { A: } 26.6 \% \\
\text { D: } 61.9 \% \\
\text { S: } 57.05 \%\end{array}$ & $\begin{array}{l}\text { A: Gender, age, } \\
\text { education, } \\
\text { family income, } \\
\text { residence, } \\
\text { family size, } \\
\text { negative } \\
\text { COVID-19 } \\
\text { perceptions, } \\
\text { and COVID-19 } \\
\text { related } \\
\text { symptoms and } \\
\text { social } \\
\text { stressors } \\
\text { D: Gender, age, } \\
\text { education, } \\
\text { family income, } \\
\text { residence, } \\
\text { family size, } \\
\text { under } 5 \\
\text { children in the } \\
\text { family, and } \\
\text { negative } \\
\text { CoVID-19 } \\
\text { perceptions } \\
\text { S: Gender, age, } \\
\text { under-five } \\
\text { children in the } \\
\text { family, } \\
\text { negative } \\
\text { COVID-19 } \\
\text { perceptions, } \\
\text { and CovID-19 } \\
\text { related } \\
\text { symptoms and } \\
\text { social } \\
\text { stressors }\end{array}$ & $\begin{array}{l}\text { Mild to } \\
\text { extremely } \\
\text { severe }\end{array}$ \\
\hline $\begin{array}{l}\text { Banna et } \\
\text { al. }(2020) \\
(20)\end{array}$ & $\begin{array}{l}\text { Cross- } \\
\text { sectional; } \\
\text { Convenience } \\
\text { \& snowball }\end{array}$ & $\begin{array}{l}\text { Online } \\
\text { survey; } \\
\text { April 29- } \\
\text { May 7, } \\
2020\end{array}$ & 1,427 & $\begin{array}{l}\text { General } \\
\text { population; } \\
\text { Age: } 25.75 \pm \\
6.75 \text { y }\end{array}$ & $\begin{array}{l}\text { DASS-21; } \\
\text { Cutoff: } \\
\text { anxiety > 6, } \\
\text { depression } \\
>9, \text { stress > } \\
10\end{array}$ & $\begin{array}{l}\text { A: } 33.7 \% \\
\text { D: } 57.9 \% \\
\text { S: } 59.7 \%\end{array}$ & $\begin{array}{l}\text { A: Gender, age, } \\
\text { marital status, } \\
\text { occupation, } \\
\text { education, } \\
\text { residence, } \\
\text { family size, } \\
\text { elderly > 50 y } \\
\text { in family and } \\
\text { negative } \\
\text { COVID-19 } \\
\text { perceptions } \\
\text { D: Gender, age, } \\
\text { marital status, } \\
\text { education, } \\
\text { elderly > 50 y } \\
\text { in family, and } \\
\text { negative } \\
\text { CoVID-19 } \\
\text { perceptions } \\
\text { s: Gender, } \\
\text { marital status, } \\
\text { occupation, } \\
\text { education, } \\
\text { family income, } \\
\text { and negative } \\
\text { COVID-19 } \\
\text { perceptions }\end{array}$ & $\begin{array}{l}\text { Mild to } \\
\text { extremely } \\
\text { severe }\end{array}$ \\
\hline
\end{tabular}




\begin{tabular}{|c|c|c|c|c|c|c|c|c|}
\hline $\begin{array}{l}\text { Authors } \\
\text { and } \\
\text { publication } \\
\text { year }\end{array}$ & $\begin{array}{l}\text { Study } \\
\text { design and } \\
\text { Sampling } \\
\text { procedure }\end{array}$ & $\begin{array}{l}\text { Data } \\
\text { collection } \\
\text { method } \\
\text { and time }\end{array}$ & $\begin{array}{l}\text { Sample } \\
\text { size }\end{array}$ & $\begin{array}{l}\text { Specific } \\
\text { group and } \\
\text { mean age }\end{array}$ & $\begin{array}{l}\text { Assessment } \\
\text { scale with } \\
\text { cutoff score }\end{array}$ & $\begin{array}{l}\text { Prevalence } \\
\text { rate(s) }\end{array}$ & Risk factors & $\begin{array}{l}\text { Assessment } \\
\text { criteria }\end{array}$ \\
\hline $\begin{array}{l}\text { Rahman et } \\
\text { al. (2020) } \\
\text { (8) }\end{array}$ & $\begin{array}{l}\text { Cross- } \\
\text { sectional; } \\
\text { Convenience } \\
\text { \& snowball }\end{array}$ & $\begin{array}{l}\text { Online } \\
\text { survey; } \\
\text { June 6- } \\
\text { June 27, } \\
2020\end{array}$ & 614 & $\begin{array}{l}\text { General } \\
\text { population; } \\
\text { NR }\end{array}$ & $\begin{array}{l}\text { DASS-21; } \\
\text { Cutoff: NR }\end{array}$ & $\begin{array}{l}\text { A: } 37 \% \\
\text { D: } 38.23 \% \\
\text { S: } 25.38 \%\end{array}$ & 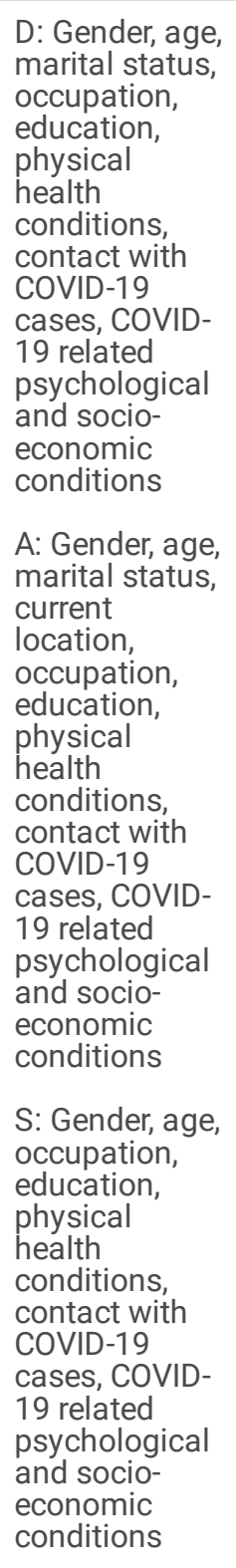 & $\begin{array}{l}\text { A \& D: } \\
\text { Moderate to } \\
\text { extremely } \\
\text { severe } \\
\text { S: Mild to } \\
\text { moderate }\end{array}$ \\
\hline $\begin{array}{l}\text { Islam et al. } \\
(2020) \\
(21)\end{array}$ & $\begin{array}{l}\text { Cross- } \\
\text { sectional; } \\
\text { NR }\end{array}$ & $\begin{array}{l}\text { Online } \\
\text { survey; } \\
\text { May 5- } \\
\text { May 15, } \\
2020\end{array}$ & 340 & $\begin{array}{l}\text { Adult } \\
\text { population; } \\
\text { Age: } 26.23 \pm \\
6.39 \mathrm{y}\end{array}$ & $\begin{array}{l}\text { Self- } \\
\text { developed } \\
\text { (single item, } \\
\text { Yes/No) }\end{array}$ & S: $85.6 \%$ & $\begin{array}{l}\text { S: Gender, age, } \\
\text { marital status, } \\
\text { and } \\
\text { occupation, } \\
\text { fear of self } \\
\text { and/or family } \\
\text { member, } \\
\text { and/or relative } \\
\text { COVID-19 } \\
\text { infection, } \\
\text { hampering } \\
\text { study and } \\
\text { career plan, } \\
\text { and financial } \\
\text { difficulties }\end{array}$ & $\begin{array}{l}\text { Binary } \\
\text { category }\end{array}$ \\
\hline
\end{tabular}




\begin{tabular}{|c|c|c|c|c|c|c|c|c|}
\hline $\begin{array}{l}\text { Authors } \\
\text { and } \\
\text { publication } \\
\text { year }\end{array}$ & $\begin{array}{l}\text { Study } \\
\text { design and } \\
\text { Sampling } \\
\text { procedure }\end{array}$ & $\begin{array}{l}\text { Data } \\
\text { collection } \\
\text { method } \\
\text { and time }\end{array}$ & $\begin{array}{l}\text { Sample } \\
\text { size }\end{array}$ & $\begin{array}{l}\text { Specific } \\
\text { group and } \\
\text { mean age }\end{array}$ & $\begin{array}{l}\text { Assessment } \\
\text { scale with } \\
\text { cutoff score }\end{array}$ & $\begin{array}{l}\text { Prevalence } \\
\text { rate(s) }\end{array}$ & Risk factors & $\begin{array}{l}\text { Assessment } \\
\text { criteria }\end{array}$ \\
\hline \multirow{2}{*}{$\begin{array}{l}\text { Barua et } \\
\text { al. }(2020) \\
(22)\end{array}$} & \multirow{2}{*}{$\begin{array}{l}\text { Cross- } \\
\text { sectional; } \\
\text { Convenience }\end{array}$} & \multirow{2}{*}{$\begin{array}{l}\text { Online } \\
\text { survey; } \\
\text { April 1- } \\
\text { May 30, } \\
2020\end{array}$} & \multirow[t]{2}{*}{370} & \multirow{2}{*}{$\begin{array}{l}\text { Frontline } \\
\text { doctors; Age: } \\
30.5 \pm 4.4 \mathrm{y}\end{array}$} & \multirow{2}{*}{$\begin{array}{l}\text { PHQ-4 (GAD } \\
2+\text { PHQ 2); } \\
\text { Cutoff: both } \\
\text { depression } \\
\text { and anxiety } \\
\geq 3\end{array}$} & A: $36.5 \%$ & \multirow[b]{2}{*}{$\begin{array}{l}\text { A and/or D: } \\
\text { Gender, } \\
\text { shifting duty, } \\
\text { inadequate } \\
\text { resources, } \\
\text { sleep } \\
\text { disturbance, } \\
\text { presence of } \\
\text { chronic } \\
\text { disease, } \\
\text { number of } \\
\text { chronic } \\
\text { conditions, } \\
\text { asthma \& } \\
\text { hypertension }\end{array}$} & \multirow{2}{*}{$\begin{array}{l}\text { Mild to } \\
\text { severe }\end{array}$} \\
\hline & & & & & & D: $38.4 \%$ & & \\
\hline \multirow{2}{*}{$\begin{array}{l}\text { Sultana et } \\
\text { al. }(2020) \\
(14)\end{array}$} & \multirow{2}{*}{$\begin{array}{l}\text { Cross- } \\
\text { sectional; } \\
\text { Convenience } \\
\text { sampling }\end{array}$} & \multirow{2}{*}{$\begin{array}{l}\text { Online } \\
\text { survey; } \\
20 \text { May- } \\
30 \text { May, } \\
2020\end{array}$} & \multirow[t]{2}{*}{707} & \multirow{2}{*}{$\begin{array}{l}\text { Wage earners; } \\
\text { Age: } 31.41 \pm \\
8.73 \text { y }\end{array}$} & PHQ-9 \& & A: $58.6 \%$ & \multirow[b]{2}{*}{$\begin{array}{l}\text { Both A and D: } \\
\text { Gender, } \\
\text { education, } \\
\text { occupation, } \\
\text { family income, } \\
\text { and current } \\
\text { financial } \\
\text { situation (e.g., } \\
\text { not getting any } \\
\text { salary, no } \\
\text { earning } \\
\text { source, salary } \\
\text { not enough for } \\
\text { family, not } \\
\text { satisfied with } \\
\text { earning, } \\
\text { upcoming } \\
\text { financial crisis, } \\
\text { children's } \\
\text { education loss, } \\
\text { increased price } \\
\text { of daily } \\
\text { necessary } \\
\text { commodities, } \\
\text { inadequate } \\
\text { food supply } \\
\text { etc.) }\end{array}$} & \multirow{2}{*}{$\begin{array}{l}\text { Moderate to } \\
\text { severe }\end{array}$} \\
\hline & & & & & $\begin{array}{l}\text { GAD-7; } \\
\text { Cutoff: } \\
\text { anxiety } \geq \\
10, \\
\text { depression } \\
\geq 10\end{array}$ & D:55.9\% & & \\
\hline \multirow{2}{*}{$\begin{array}{l}\text { Ripon et al. } \\
\text { (2020) } \\
(23)\end{array}$} & \multirow{2}{*}{$\begin{array}{l}\text { Cross- } \\
\text { sectional; } \\
\text { NR }\end{array}$} & \multirow{2}{*}{$\begin{array}{l}\text { Online } \\
\text { survey; } \\
\text { till } \\
\text { October } \\
19,2020\end{array}$} & \multirow[t]{2}{*}{5792} & \multirow{2}{*}{$\begin{array}{l}\text { Adult } \\
\text { quarantine } \\
\text { population; } \\
\text { Age: NR }\end{array}$} & \multirow{2}{*}{$\begin{array}{l}\text { CES-D \& } \\
\text { IES-R; } \\
\text { Cutoff: } \\
\text { depression } \\
\geq 16 \text {, PTSD } \\
\geq 20\end{array}$} & D: $24 \%$ & \multirow[b]{2}{*}{$\begin{array}{l}\text { PTSD: Socio- } \\
\text { demographic } \\
\text { risk factors are } \\
\text { not reported. } \\
\text { Nutritional } \\
\text { behavior } \\
\text { changes, the } \\
\text { likelihood of } \\
\text { infection, } \\
\text { nutritional } \\
\text { perceived } \\
\text { efficiency, } \\
\text { exaggeration } \\
\text { of risk, eating } \\
\text { or taking } \\
\text { substances, } \\
\text { the severity of } \\
\text { the fear. }\end{array}$} & \multirow{2}{*}{$\begin{array}{l}\text { Standard } \\
\text { cutoff } \\
\text { points }\end{array}$} \\
\hline & & & & & & $\begin{array}{l}\text { PTSD: } \\
35 \%\end{array}$ & & \\
\hline
\end{tabular}




\begin{tabular}{|c|c|c|c|c|c|c|c|c|}
\hline $\begin{array}{l}\text { Authors } \\
\text { and } \\
\text { publication } \\
\text { year }\end{array}$ & $\begin{array}{l}\text { Study } \\
\text { design and } \\
\text { Sampling } \\
\text { procedure }\end{array}$ & $\begin{array}{l}\text { Data } \\
\text { collection } \\
\text { method } \\
\text { and time }\end{array}$ & $\begin{array}{l}\text { Sample } \\
\text { size }\end{array}$ & $\begin{array}{l}\text { Specific } \\
\text { group and } \\
\text { mean age }\end{array}$ & $\begin{array}{l}\text { Assessment } \\
\text { scale with } \\
\text { cutoff score }\end{array}$ & $\begin{array}{l}\text { Prevalence } \\
\text { rate(s) }\end{array}$ & Risk factors & $\begin{array}{l}\text { Assessment } \\
\text { criteria }\end{array}$ \\
\hline $\begin{array}{l}\text { Hasan et } \\
\text { al. (2020) } \\
(24)\end{array}$ & $\begin{array}{l}\text { Cross- } \\
\text { sectional; } \\
\text { Convenience } \\
\text { sampling }\end{array}$ & $\begin{array}{l}\text { Online } \\
\text { survey; } \\
\text { April 21- } \\
\text { May 10, } \\
2020\end{array}$ & 412 & $\begin{array}{l}\text { Physicians; } \\
\text { NR }\end{array}$ & $\begin{array}{l}\text { HADS; } \\
\text { Cutoff: } \\
\text { higher score } \\
\text { indicates } \\
\text { higher level } \\
\text { of } \\
\text { symptoms }\end{array}$ & $\begin{array}{l}\text { A: } 67.72 \% \\
\text { D: } 48.5 \%\end{array}$ & $\begin{array}{l}\text { A and/or D: } \\
\text { Gender, } \\
\text { experienced } \\
\text { symptoms of } \\
\text { COVID-19, had } \\
\text { not received } \\
\text { incentives/ } \\
\text { just } \\
\text { commitments, } \\
\text { self-funded } \\
\text { source of PPE, } \\
\text { inadequate } \\
\text { training, } \\
\text { lacking } \\
\text { perceived self- } \\
\text { efficacy to } \\
\text { control coVID } \\
\text { - } 19 \text { positive } \\
\text { patients, } \\
\text { severe tension } \\
\text { of getting } \\
\text { infected with } \\
\text { COVID-19, } \\
\text { extreme } \\
\text { tension about } \\
\text { family } \\
\text { members } \\
\text { getting } \\
\text { infected with } \\
\text { coVID-19, } \\
\text { check daily } \\
\text { news/update } \\
\text { more than or } \\
\text { equal to } 4 \\
\text { times, } \\
\text { difficulty to } \\
\text { stay away } \\
\text { from media, } \\
\text { hard to stay } \\
\text { away from } \\
\text { social media, } \\
\text { daily leisure } \\
\text { time less than } \\
2 \text { hours, didn't } \\
\text { earn enough to } \\
\text { support the } \\
\text { family during } \\
\text { the pandemic, } \\
\text { afraid of } \\
\text { getting } \\
\text { assaulted on } \\
\text { the way to } \\
\text { home or } \\
\text { hospital, sleep } \\
\text { disturbances, } \\
\text { being agitated } \\
\text { more usually. }\end{array}$ & NR \\
\hline
\end{tabular}




\begin{tabular}{|c|c|c|c|c|c|c|c|c|}
\hline $\begin{array}{l}\text { Authors } \\
\text { and } \\
\text { publication } \\
\text { year }\end{array}$ & $\begin{array}{l}\text { Study } \\
\text { design and } \\
\text { Sampling } \\
\text { procedure }\end{array}$ & $\begin{array}{l}\text { Data } \\
\text { collection } \\
\text { method } \\
\text { and time }\end{array}$ & $\begin{array}{l}\text { Sample } \\
\text { size }\end{array}$ & $\begin{array}{l}\text { Specific } \\
\text { group and } \\
\text { mean age }\end{array}$ & $\begin{array}{l}\text { Assessment } \\
\text { scale with } \\
\text { cutoff score }\end{array}$ & $\begin{array}{l}\text { Prevalence } \\
\text { rate(s) }\end{array}$ & Risk factors & $\begin{array}{l}\text { Assessment } \\
\text { criteria }\end{array}$ \\
\hline $\begin{array}{l}\text { Tasnim et } \\
\text { al. }(2020) \\
(25)\end{array}$ & $\begin{array}{l}\text { Cross- } \\
\text { sectional; } \\
\text { NR }\end{array}$ & $\begin{array}{l}\text { Online } \\
\text { survey; } \\
\text { between } \\
\text { July and } \\
\text { August, } \\
2020\end{array}$ & 803 & $\begin{array}{l}\text { Healthcare } \\
\text { professionals; } \\
\text { Age: } 27.3 \pm \\
6.9 \text { y }\end{array}$ & $\begin{array}{l}\text { HADS, (both } \\
\text { depression } \\
\text { and anxiety } \\
\geq 8 \text { ) }\end{array}$ & $\begin{array}{l}\text { A: } 69.5 \% \\
\text { D: } 39.5 \%\end{array}$ & $\begin{array}{l}\text { A and D: } \\
\text { Gender, having } \\
\text { moderate and } \\
\text { poor health } \\
\text { status, } \\
\text { irregular } \\
\text { physical } \\
\text { exercise, } \\
\text { smoking, } \\
\text { having regrets } \\
\text { about their } \\
\text { profession } \\
\text { because of the } \\
\text { pandemic and } \\
\text { many } \\
\text { unexpected } \\
\text { experiences, } \\
\text { experiencing } \\
\text { discrimination } \\
\text { in the } \\
\text { workplace, and } \\
\text { facing social } \\
\text { problems due } \\
\text { to working in a } \\
\text { lab or hospital. }\end{array}$ & $\begin{array}{l}\text { Borderline } \\
\text { abnormal to } \\
\text { abnormal }\end{array}$ \\
\hline $\begin{array}{l}\text { Ahmed et } \\
\text { al. (2020) } \\
(26)\end{array}$ & $\begin{array}{l}\text { Cross- } \\
\text { sectional; } \\
\text { NR }\end{array}$ & $\begin{array}{l}\text { Online } \\
\text { survey; } \\
2020\end{array}$ & 500 & $\begin{array}{l}\text { General } \\
\text { population; } \\
\text { Age: } 25.95 \pm \\
7.57 \mathrm{y}\end{array}$ & $\begin{array}{l}\text { DASS-21; } \\
\text { Cutoff: } \\
\text { anxiety } \geq 4, \\
\text { depression } \\
\geq 5 \text {, stress } \\
\geq 8\end{array}$ & $\begin{array}{l}\text { A: } 39.8 \% \\
\text { D: } 43 \% \\
\text { S: } 34.8 \%\end{array}$ & $\begin{array}{l}\text { D, A and S: } \\
\text { Gender, } \\
\text { educational } \\
\text { level, } \\
\text { occupation, } \\
\text { marital status, } \\
\text { and age. }\end{array}$ & $\begin{array}{l}\text { Mild to } \\
\text { extremely } \\
\text { severe }\end{array}$ \\
\hline $\begin{array}{l}\text { Hossain et } \\
\text { al. (2020) } \\
\text { (27) }\end{array}$ & $\begin{array}{l}\text { Cross- } \\
\text { sectional; } \\
\text { NR }\end{array}$ & $\begin{array}{l}\text { Online } \\
\text { survey; } \\
\text { 3rd week } \\
\text { of March, } \\
2020\end{array}$ & 880 & $\begin{array}{l}\text { General } \\
\text { population; } \\
\text { Age: } 26.3 \pm \\
7.2 \text { y }\end{array}$ & $\begin{array}{l}\text { GAD-7; } \\
\text { Cutoff: } \\
\text { anxiety } \geq \\
10\end{array}$ & A: $49.1 \%$ & $\begin{array}{l}\text { A: Social } \\
\text { media } \\
\text { exposure over } \\
4 \text { hours a day, } \\
\text { bad health } \\
\text { condition and } \\
\text { self-rated } \\
\text { health }\end{array}$ & NR \\
\hline $\begin{array}{l}\text { Islam et al. } \\
(2020) \\
(28)\end{array}$ & $\begin{array}{l}\text { Cross- } \\
\text { sectional; } \\
\text { Snowball }\end{array}$ & $\begin{array}{l}\text { Online } \\
\text { survey; } \\
\text { May 6- } \\
\text { May 12, } \\
2020\end{array}$ & 476 & $\begin{array}{l}\text { University } \\
\text { students; Age: } \\
\text { NR }\end{array}$ & $\begin{array}{l}\text { GAD-7 \& } \\
\text { PHQ-9; } \\
\text { Cutoff: } \\
\text { anxiety } \geq 5 \text {, } \\
\text { depression } \\
\geq 5\end{array}$ & $\begin{array}{l}\text { A: } 81.8 \% \\
\text { D: } 82.4 \%\end{array}$ & $\begin{array}{l}\text { D and A: } \\
\text { private tuition } \\
\text { during COVID- } \\
19 \text {, lagging } \\
\text { academically, } \\
\text { living with } \\
\text { family }\end{array}$ & $\begin{array}{l}\text { Mild to } \\
\text { extremely } \\
\text { severe }\end{array}$ \\
\hline $\begin{array}{l}\text { Islam et al. } \\
\text { (2020a) } \\
(29)\end{array}$ & $\begin{array}{l}\text { Cross- } \\
\text { sectional; } \\
\text { NR }\end{array}$ & $\begin{array}{l}\text { Online } \\
\text { survey; } \\
\text { March } \\
\text { 29-April } \\
\text { 6, 2020 }\end{array}$ & 1311 & $\begin{array}{l}\text { General } \\
\text { population; } \\
\text { Age: } 23.54 \pm \\
4.97 \mathrm{y}\end{array}$ & $\begin{array}{l}\text { GAD-7; } \\
\text { Cutoff: } \\
\text { anxiety } \geq \\
10\end{array}$ & A: $37.3 \%$ & $\begin{array}{l}\text { A: Gender, age, } \\
\text { education } \\
\text { level, married, } \\
\text { occupation, } \\
\text { panic }\end{array}$ & $\begin{array}{l}\text { Moderate to } \\
\text { extremely } \\
\text { severe }\end{array}$ \\
\hline $\begin{array}{l}\text { Mamun et } \\
\text { al. (2020) } \\
(30)\end{array}$ & $\begin{array}{l}\text { Cross- } \\
\text { sectional; } \\
\text { NR }\end{array}$ & $\begin{array}{l}\text { Online } \\
\text { survey; } \\
\text { April 1- } \\
\text { April 10, } \\
2020\end{array}$ & 10067 & $\begin{array}{l}\text { General } \\
\text { population; } \\
\text { Age: } 29.9 \pm \\
9.6 \text { y }\end{array}$ & $\begin{array}{l}\text { PHQ-9; } \\
\text { Cutoff: } \\
\text { depression } \\
\geq 10\end{array}$ & D:33.3\% & $\begin{array}{l}\text { D: Gender, age, } \\
\text { smoking, } \\
\text { having } \\
\text { comorbid } \\
\text { diseases and } \\
\text { insomnia, } \\
\text { having high } \\
\text { scores on the } \\
\text { Fear COVID-19 } \\
\text { Scale. }\end{array}$ & NR \\
\hline
\end{tabular}




\begin{tabular}{|c|c|c|c|c|c|c|c|c|}
\hline $\begin{array}{l}\text { Authors } \\
\text { and } \\
\text { publication } \\
\text { year }\end{array}$ & $\begin{array}{l}\text { Study } \\
\text { design and } \\
\text { Sampling } \\
\text { procedure }\end{array}$ & $\begin{array}{l}\text { Data } \\
\text { collection } \\
\text { method } \\
\text { and time }\end{array}$ & $\begin{array}{l}\text { Sample } \\
\text { size }\end{array}$ & $\begin{array}{l}\text { Specific } \\
\text { group and } \\
\text { mean age }\end{array}$ & $\begin{array}{l}\text { Assessment } \\
\text { scale with } \\
\text { cutoff score }\end{array}$ & $\begin{array}{l}\text { Prevalence } \\
\text { rate(s) }\end{array}$ & Risk factors & $\begin{array}{l}\text { Assessment } \\
\text { criteria }\end{array}$ \\
\hline \multirow{3}{*}{$\begin{array}{l}\text { Al Zubayer } \\
\text { et al. } \\
(2020) \\
(31)\end{array}$} & \multirow{3}{*}{$\begin{array}{l}\text { Cross- } \\
\text { sectional; } \\
\text { NR }\end{array}$} & \multirow{3}{*}{$\begin{array}{l}\text { Online } \\
\text { survey; } \\
\text { June 1- } \\
\text { June 10, } \\
2020\end{array}$} & \multirow[t]{3}{*}{1146} & \multirow{3}{*}{$\begin{array}{l}\text { General } \\
\text { population; } \\
\text { Age: } 26.38 \pm \\
8.87 \mathrm{y}\end{array}$} & \multirow{3}{*}{$\begin{array}{l}\text { DASS-21; } \\
\text { Cutoff: } \\
\text { anxiety } \geq \\
10 \\
\text { depression } \\
\geq 14 \text {, stress } \\
\geq 19\end{array}$} & & \multirow{3}{*}{$\begin{array}{l}\text { D, A and S: } \\
\text { Following } \\
\text { COVID-19 } \\
\text { related news } \\
\text { daily, having } \\
\text { COVID-19 } \\
\text { symptoms, } \\
\text { having direct } \\
\text { and indirect } \\
\text { contact with } \\
\text { COVID-19 } \\
\text { infected } \\
\text { people, fear of } \\
\text { COVID-19 } \\
\text { infection. }\end{array}$} & \multirow{3}{*}{$\begin{array}{l}\text { Moderate to } \\
\text { extremely } \\
\text { severe }\end{array}$} \\
\hline & & & & & & D:47.2\% & & \\
\hline & & & & & & S: $32.5 \%$ & & \\
\hline \multirow{3}{*}{$\begin{array}{l}\text { Safa et al. } \\
(2021) \\
(32)\end{array}$} & \multirow{3}{*}{$\begin{array}{l}\text { Cross- } \\
\text { sectional; } \\
\text { Convenience } \\
\& \text { snowball }\end{array}$} & \multirow{3}{*}{$\begin{array}{l}\text { Online } \\
\text { survey; } \\
\text { April } 21 \\
\text { to May } \\
10,2020\end{array}$} & \multirow[t]{3}{*}{425} & \multirow{3}{*}{$\begin{array}{l}\text { Medical } \\
\text { students; } \\
\text { Age: } 22 \pm 1.81 \\
\text { y }\end{array}$} & \multirow{3}{*}{$\begin{array}{l}\text { HADS; } \\
\text { Cutoff: both } \\
\text { depression } \\
\text { and anxiety } \\
>8\end{array}$} & \multirow{3}{*}{$\begin{array}{l}\text { A: } 65.9 \% \\
\text { D: } 49.9 \%\end{array}$} & \multirow[b]{2}{*}{$\begin{array}{l}\text { A: Fear of } \\
\text { getting } \\
\text { infected with } \\
\text { COVID-19, fear } \\
\text { of getting } \\
\text { assaulted or } \\
\text { humiliated on } \\
\text { the way to } \\
\text { hospital or } \\
\text { home, lack of } \\
\text { concentration } \\
\text { on the study, } \\
\text { agitation. }\end{array}$} & \multirow[t]{3}{*}{$\begin{array}{l}\text { Mild to } \\
\text { severe }\end{array}$} \\
\hline & & & & & & & & \\
\hline & & & & & & & $\begin{array}{l}\text { D: Gender, fear } \\
\text { of getting } \\
\text { infected with } \\
\text { COVID-19, } \\
\text { agitation. }\end{array}$ & \\
\hline
\end{tabular}




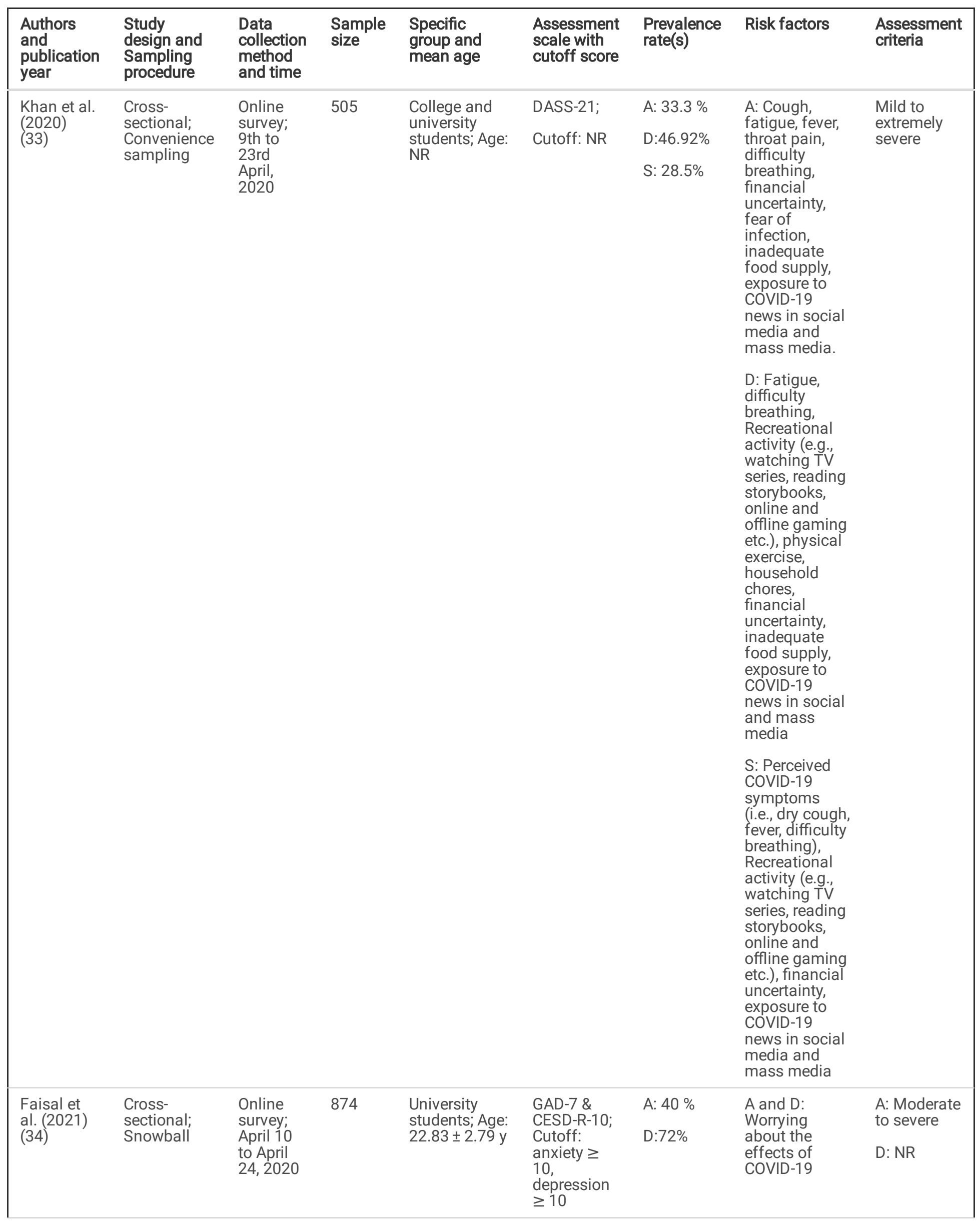




\begin{tabular}{|c|c|c|c|c|c|c|c|c|}
\hline $\begin{array}{l}\text { Authors } \\
\text { and } \\
\text { publication } \\
\text { year }\end{array}$ & $\begin{array}{l}\text { Study } \\
\text { design and } \\
\text { Sampling } \\
\text { procedure }\end{array}$ & $\begin{array}{l}\text { Data } \\
\text { collection } \\
\text { method } \\
\text { and time }\end{array}$ & $\begin{array}{l}\text { Sample } \\
\text { size }\end{array}$ & $\begin{array}{l}\text { Specific } \\
\text { group and } \\
\text { mean age }\end{array}$ & $\begin{array}{l}\text { Assessment } \\
\text { scale with } \\
\text { cutoff score }\end{array}$ & $\begin{array}{l}\text { Prevalence } \\
\text { rate(s) }\end{array}$ & Risk factors & $\begin{array}{l}\text { Assessment } \\
\text { criteria }\end{array}$ \\
\hline $\begin{array}{l}\text { Islam et al. } \\
\text { (2020b) } \\
(35)\end{array}$ & $\begin{array}{l}\text { Cross- } \\
\text { sectional; } \\
\text { Convenience } \\
\text { sampling }\end{array}$ & $\begin{array}{l}\text { Online } \\
\text { survey; } \\
\text { during } \\
\text { April } \\
2020\end{array}$ & 3,122 & $\begin{array}{l}\text { University } \\
\text { students; Age: } \\
21.4 \pm 2 \text { y }\end{array}$ & $\begin{array}{l}\text { DASS-21; } \\
\text { Cutoff: } \\
\text { anxiety } \geq 8, \\
\text { depression } \\
\geq 10 \text {, stress } \\
\geq 15\end{array}$ & $\begin{array}{l}\text { A: } 71.5 \% \\
\text { D: } 76.1 \% \\
\text { S: } 70.1 \%\end{array}$ & $\begin{array}{l}\text { Both D, A, and } \\
\text { S: Gender, } \\
\text { being older } \\
\text { ( } 25-29 \text { years), } \\
\text { having } \geq 5 \\
\text { family } \\
\text { members, } \\
\text { living in urban } \\
\text { areas, not } \\
\text { engaging in } \\
\text { physical } \\
\text { exercise, } \\
\text { having } \\
\text { dissatisfaction } \\
\text { with their } \\
\text { sleep, } \\
\text { spending more } \\
\text { hours } \\
\text { browsing the } \\
\text { internet, } \\
\text { having } \\
\text { dissatisfaction } \\
\text { with academic } \\
\text { studies under } \\
\text { the present } \\
\text { COVID-19 } \\
\text { circumstances, } \\
\text { and smoking }\end{array}$ & $\begin{array}{l}\text { At least } \\
\text { mild } \\
\text { symptoms }\end{array}$ \\
\hline $\begin{array}{l}\text { Sakib et al. } \\
(2021) \\
(36)\end{array}$ & $\begin{array}{l}\text { Cross- } \\
\text { sectional; } \\
\text { NR }\end{array}$ & $\begin{array}{l}\text { Online } \\
\text { survey; } \\
\text { between } \\
\text { April } 8 \text { to } \\
\text { April 25, } \\
2020\end{array}$ & 3,388 & $\begin{array}{l}\text { General } \\
\text { population; } \\
\text { Age: } 30.11 \pm \\
6.44 \mathrm{y}\end{array}$ & $\begin{array}{l}\text { PHQ-9; } \\
\text { Cutoff: } \\
\text { depression } \\
\geq 10\end{array}$ & D: $27.8 \%$ & $\begin{array}{l}\text { Gender, marital } \\
\text { status, and } \\
\text { having chronic } \\
\text { physical } \\
\text { diseases, } \\
\text { having } \\
\text { children }\end{array}$ & NR \\
\hline $\begin{array}{l}\text { Mina et al. } \\
(2021) \\
(37)\end{array}$ & $\begin{array}{l}\text { Cross- } \\
\text { sectional; } \\
\text { NR }\end{array}$ & $\begin{array}{l}\text { Online } \\
\text { survey; } \\
\text { May } 26 \\
\text { to June } \\
27,2020\end{array}$ & 153 & $\begin{array}{l}\text { COVID-19 } \\
\text { infected } \\
\text { people; } \\
\text { Age: } 39.43 \pm \\
17.59 \mathrm{y}\end{array}$ & $\begin{array}{l}\text { GAD-7 \& } \\
\text { PHQ-9; } \\
\text { Cutoff: both } \\
\text { anxiety \& } \\
\text { depression } \\
\geq 10\end{array}$ & $\begin{array}{l}\text { A: } 63.5 \% \\
\text { D: } 56.6 \%\end{array}$ & NR & $\begin{array}{l}\text { Moderate to } \\
\text { extremely } \\
\text { severe }\end{array}$ \\
\hline $\begin{array}{l}\text { Dhar et al. } \\
(2020) \\
(38)\end{array}$ & $\begin{array}{l}\text { Cross- } \\
\text { sectional; } \\
\text { NR }\end{array}$ & NR & 15,543 & $\begin{array}{l}\text { University } \\
\text { students; Age: } \\
\text { NR }\end{array}$ & $\begin{array}{l}\text { GAD-7; } \\
\text { Cutoff: NR }\end{array}$ & $\begin{array}{l}\text { A: Mild } \\
3.82 \% \text {, } \\
\text { Moderate } \\
\text { 48.41\%, } \\
\text { Severe: } \\
\text { 44.59\% }\end{array}$ & $\begin{array}{l}\text { A: Gender, } \\
\text { living in the } \\
\text { urban area, } \\
\text { living with } \\
\text { their parents, } \\
\text { financial } \\
\text { condition, } \\
\text { infection of } \\
\text { COVID-19 } \\
\text { among } \\
\text { relatives or } \\
\text { friends }\end{array}$ & NR \\
\hline
\end{tabular}




\begin{tabular}{|c|c|c|c|c|c|c|c|c|}
\hline $\begin{array}{l}\text { Authors } \\
\text { and } \\
\text { publication } \\
\text { year }\end{array}$ & $\begin{array}{l}\text { Study } \\
\text { design and } \\
\text { Sampling } \\
\text { procedure }\end{array}$ & $\begin{array}{l}\text { Data } \\
\text { collection } \\
\text { method } \\
\text { and time }\end{array}$ & $\begin{array}{l}\text { Sample } \\
\text { size }\end{array}$ & $\begin{array}{l}\text { Specific } \\
\text { group and } \\
\text { mean age }\end{array}$ & $\begin{array}{l}\text { Assessment } \\
\text { scale with } \\
\text { cutoff score }\end{array}$ & $\begin{array}{l}\text { Prevalence } \\
\text { rate(s) }\end{array}$ & Risk factors & $\begin{array}{l}\text { Assessment } \\
\text { criteria }\end{array}$ \\
\hline $\begin{array}{l}\text { Yasmin et } \\
\text { al. (2021) } \\
(39)\end{array}$ & $\begin{array}{l}\text { Cross- } \\
\text { sectional; } \\
\text { Purposive }\end{array}$ & $\begin{array}{l}\text { Online } \\
\text { survey; } \\
\text { June 17 } \\
\text { to June } \\
25,2020\end{array}$ & 248 & $\begin{array}{l}\text { Bankers; } \\
\text { Age: NR }\end{array}$ & $\begin{array}{l}\text { DASS-21; } \\
\text { Cutoff: } \\
\text { anxiety, } \\
\text { depression, } \\
\text { stress } \geq 21\end{array}$ & $\begin{array}{l}\text { A: } 10.6 \% \\
\text { D: } 12.1 \% \\
\text { S: } 11.1 \%\end{array}$ & $\begin{array}{l}\text { S: Gender, } \\
\text { relationship } \\
\text { status, having } \\
\text { an older adult } \\
\text { and a } \\
\text { pregnant } \\
\text { woman at } \\
\text { home, having } \\
\text { fair knowledge } \\
\text { on COVID-19, } \\
\text { colleagues } \\
\text { infected by } \\
\text { COVID-19, } \\
\text { smoking more } \\
\text { frequently, } \\
\text { having a bad } \\
\text { dream during } \\
\text { sleep. } \\
\text { A: Living with } \\
\text { relatives, } \\
\text { colleagues } \\
\text { infected by } \\
\text { COVID-19, } \\
\text { using public } \\
\text { transportation, } \\
\text { having a bad } \\
\text { dream during } \\
\text { sleep, } \\
\text { misbehaving } \\
\text { with family } \\
\text { members and } \\
\text { beaten } \\
\text { children. } \\
\text { D: Living with } \\
\text { relatives, } \\
\text { having an } \\
\text { elderly person } \\
\text { and a } \\
\text { pregnant } \\
\text { woman at } \\
\text { home, fear of } \\
\text { getting } \\
\text { infected, } \\
\text { smoking more } \\
\text { frequently, } \\
\text { having a bad } \\
\text { dream during } \\
\text { sleep. }\end{array}$ & $\begin{array}{l}\text { Severely to } \\
\text { extremely }\end{array}$ \\
\hline $\begin{array}{l}\text { Khatun et } \\
\text { al. (2021) } \\
(40)\end{array}$ & $\begin{array}{l}\text { Cross- } \\
\text { sectional; } \\
\text { NR }\end{array}$ & $\begin{array}{l}\text { Online } \\
\text { survey; } \\
\text { May } 4 \text { to } \\
\text { May } 10 \text {, } \\
2020\end{array}$ & 114 & $\begin{array}{l}\text { Physicians; } \\
\text { Age: NR }\end{array}$ & $\begin{array}{l}\text { GAD-7 \& } \\
\text { PHQ-9; } \\
\text { Cutoff: } \\
\text { anxiety } \geq 9 \\
\text { depression } \\
\geq 10\end{array}$ & $\begin{array}{l}\text { A: } 32.5 \% \\
\text { D: } 34.2 \%\end{array}$ & $\begin{array}{l}\text { A: Work per } \\
\text { day ( } 8 \text { hours or } \\
\text { more) and } \\
\text { current job } \\
\text { location } \\
\text { (Dhaka } \\
\text { division) }\end{array}$ & NR \\
\hline $\begin{array}{l}\text { A - Anxiety; } \\
\text { Depression } \\
\text { Epidemiolog } \\
\text { Revised }\end{array}$ & $\begin{array}{l}\text { - Depressio } \\
\text { ale; DASS-21 } \\
\text { studies- Dep }\end{array}$ & $\begin{array}{l}\text { - Stress; } \mathbf{P} \\
\text { epression, } \\
\text { sion; MDD: }\end{array}$ & $\begin{array}{l}\text { D - Pos } \\
\text { kiety, Stre } \\
\text { ajor Depr }\end{array}$ & $\begin{array}{l}\text { Traumatic Str } \\
\text { s Scale 21; IES } \\
\text { ssive Disorder }\end{array}$ & $\begin{array}{l}\text { isorders; NR: } \\
\text { mpact of ever } \\
\text { D-R-10: Cente }\end{array}$ & $\begin{array}{l}\text { ot Reported; } \\
\text { scale-Revise } \\
\text { for Epidemio }\end{array}$ & $\begin{array}{l}\text { ADS - Hospital } \\
\text {; CES-D: Center f } \\
\text { gic Studies Dep }\end{array}$ & $\begin{array}{l}\text { xiety and } \\
\text { ssion Scale- }\end{array}$ \\
\hline
\end{tabular}

\section{Results}


A total of 24 studies were encompassed in the present review after confirming the aforementioned inclusion criteria, whereas a total of 49,806 respondents participated autonomously, including a range of sample size 114 to 15,543 ; whereas the mean age of the participants ranged between $21.4( \pm 2)$ and $31.41( \pm 8.73)$ years. All of the included studies were cross-sectional and were conducted through online media and platforms. Most of the studies were surveyed among general population $(n=9)$ including different cohorts e.g., students $(n=7)$, healthcare professionals $(n=4)$, quarantined individuals $(n=1)$, wage-earners $(n=1)$, SARS-CoV- 2 infected individuals $(n=1)$, and bankers $(n=1)$. In addition, utmost of the participants belonged to younger adult age groups. Different diagnostic tools were used to detect mental health problems, whereas most of the studies utilized the DASS-21 $(n=7)$. There are also disparities in the prevalence cutoff values; that is, some of the studies $(n=8)$ considered 'mild to severe or extremely severe conditions' for identifying prevalence rates, whereas 'moderate to severe or extremely severe' in 6 studies, and nine studies did not report (or self-considered) the cutoff points. The overall description of the recruited articles can be found in Table 1.

Distribution of Mental Disorders across Different Cohorts

The included articles assessed the ranges of mental health problems into a total of 7 distinct groups (e.g., general population, students, healthcare professionals, bankers, quarantined people, wage earners, and SARS-CoV-2 infected people), whereas each group has shown huge fluctuation in case of the prevalence of the mental health outcomes. However, higher rates of anxiety (81.8\%) were observed among university students, whereas the lower was for bankers (10.6\%). Similarly, this rate was $82.4 \%$ among university students and $12.1 \%$ for bankers, and $85.6 \%$ among general people and $11.1 \%$ for bankers, respectively, for higher and lower prevalence rates of depression and stress. Surprisingly, all of the lower rates of mental health outcomes were observed among bankers.

a. Prevalence of mental disorders: General population

As of observing a total of 9 studies, the prevalence rate of anxiety, depression and stress among the general population ranged from $33.7-49.1 \%, 27.8-57.9 \%$, and $25.38-85.6 \%$, respectively $(8,20,21,26,27,29-31,36)$. General people experience a huge fluctuation of mental disorders since people belonging to various socio-economic statuses like students, healthcare professionals, business workers, wage-earners, retired people, unemployed, farmers, homemakers, etc. Most of the studies conducted among general population prevalence rates mostly included student participants as being of higher availability to online study.

b. Prevalence of mental disorders: Students

A total of 7 studies reported mental disorders among students, where the prevalence rates ranged from $46.92-82.4 \%, 26.6-81.8 \%$ and $28.5-70.1 \%$, respectively, for depression, anxiety and stress $(19,28,32-35,41)$. All of the studies were conducted during the month of April and May in 2020, where studies reported the rate had shown a considerable fluctuation, which might be due to the utilization of separate cutoff scores along with separate assessment tools.

c. Prevalence of mental disorders: Healthcare professionals

Due to close contact with the COVID-19 infected and/or suspected patients, the healthcare professionals generally experience higher psychological distresses. Only four studies were conducted focusing on the mental health of the healthcare professionals, whereas the prevalence of depression and anxiety ranged from $34.2-48.5 \%$ and $32.5-69.5 \%$, respectively $(22,24,25,40)$. Nevertheless, stress was not reported in any of the studies.

d. Prevalence of mental disorders: Bankers

During the COVID-19 pandemic, banks were not closed down, which might influence the bankers attaining higher mental health problems as they had to go to banks through public transportation mostly. Therefore, fear of being infected also increases their risk of being affected mentally. Nevertheless, only a study was directed among bankers regarding the level of anxiety, depression and stress, which includes $10.6 \%, 12.1 \%$ and $11.1 \%$, respectively; the lowest prevalence rates as identified across all of the cohorts included in this review (39).

e. Prevalence of mental disorders: Quarantined people

Quarantine or restriction of movement had been introduced to switch the transmission of SARS-CoV-2 during the COVID-19 pandemic, wherever strategies associated with quarantine were constantly washing hands, wearing masks, and departing to the hospital when

Page 14/23 
experiencing any complications related to COVID-19 symptoms (23). Nevertheless, these effective measures had been reported impacting psychologically on the quarantined people by evolving personalized trauma, fear and uncertainty of their natural life etc. Following the situation, only a study was conducted among the quarantined people, whereas the quarantined people reported experiencing $24 \%$ and $35 \%$ depression and PTSD, respectively (23).

f. Prevalence of mental disorders: Wage-earners

As of the COVID-19 related lockdown (e.g., movement restriction and earning losses), the wage-earning people became unable to bear the basic family needs, which effect on them being psychologically more vulnerable. Thus, as reported in a study, higher mental disorders were reported to this cohort; that is, $58.6 \%$ and $55.9 \%$ were the prevalence of anxiety and depression (14).

g. Prevalence of mental disorders: COVID-19 infected people

People infected with the COVID-19 generally experience a higher level of psychological problems, including depression and anxiety as a fear of mortality. As a vulnerable cohort, higher mental health problems were observed in this group, e.g., $63.5 \%$ and $56.6 \%$ for anxiety and depression. Hence, only a study was directed among this vulnerable cohort (37).

Risk Factors Associated with Mental Disorders

The risk factors for mental disorders can be divided into three major categories, that is, (i) socio-demographic, (ii) behavior and health, and (iii) COVID-19 related risk factors. The basic socio-demographic risk factors included age, gender, family income, marital status, education, residence and family type, and having elderly and children in the family. In contrast, smoking and alcohol use, social media exposure, physical exercise, physical health conditions, and psychological conditions were the behavior and health-related risk factors. Finally, COVID-19 related risk factors included insufficient protective resources, COVID-19 related perceptions, fear of COVID-19 infection, experiencing COVID-19 related symptoms, being suspected/infected with the COVID-19, being contacted with the COVID-19 cases, being quarantined or isolated etc.

\subsubsection{Socio-Demographic Risk Factors}

\section{a. Age}

Age had been found significant in several studies that influenced mental health consequences $(8,14,19-21,26,29,30,35,40)$. Whereas Sayeed et al. (19) found participants' age less than or equal to 22 years were at 4.49, 4.46 and 3-fold higher risk of developing depression, anxiety and stress respectively than age more than 22 years. However, another study (20) reported that being aged between 24 and 39 than $\geq 40$ years had a lower risk of being anxious, but those aged $\leq 23$ years compared to $\geq 40$ years had experienced higher depressive symptoms. Similarly, Mamun et al. (30) reported that younger people develop depressive symptoms mostly. Besides, Islam et al. (29) found that participants aged 13 to 20 and 21 to 30 years were at a lower risk of anxiety than more than 30 years. Another study reported that participants' being younger were at greater risk of developing psychological sufferings (35). Furthermore, respondents aged below 25 years were approximately 2.29 times more prone to depression suffering than those more than 35 years old (14). The included studies to this review randomly utilized different age ranges; hence, concluding age-based mental health risk factors can be limited.

b. Gender

Gender was found to be a significant predictor of mental disorders in a large number of studies $(8,14,19-22,24-26,29,30,32,35,36,38-$ 40). A study found that females were suffering mostly from depression, anxiety, and stress, respectively, where it was 3.4-, 3.4- and 4.5 times higher than males (19). Similarly, al Banna et al. (20) reported that male participants were reported significantly less vulnerable to stress, anxiety and depression. Consistent with this, other studies also confirmed that female participants were at greater risk of suffering from mental disorders $(25,26,29,30,32)$. However, no studies considered transgender concerning mental health in Bangladesh; although Mamun et al. (30) collected data on it, transgender was excluded from the analyses concerning the presentation of mental health problems.

c. Family monthly income

Only 2- studies found family income as a significant factor in psychological sufferings $(14,19)$. Participants' monthly family income $\leq$ 2,7000 BDT [Bangladeshi Taka (currency)] was at approximately 2.56- and 2.62-folds higher risk of developing anxiety and depressive symptoms, respectively, than $\geq 27,000$ BDT (19). However, another study concerning the wage-earning participants reported that the

Page $15 / 23$ 
average monthly family income to be associated with both symptoms of anxiety and depression; that is, 3.31 and 4.12 - times higher risk, respectively, were observed for less than 30,000 BDT compared to more than 70,000 BDT (14).

d. Marital status

Marital status had been found significant in a total of 6- studies $(21,26,29,36,39,40)$, which depicted that a married person was at approximately 2-times higher risk of suffering from anxiety than an unmarried person (29). But heterogeneity of depression rate distribution with marital status was also found in a comparative study among the general population and healthcare professionals; that is, the divorced participants reported the highest depression rate followed by those who were unmarried and married $(53.5 \%, 33.6 \%$, and $23.7 \%$, respectively) (36). Similarly, physicians being either unmarried or divorced or widowed or separated compared to married ones experiencing 3 - times higher depressive symptoms (40).

e. Education

Education has been a significant factor in mental disorders in some of the studies $(8,14,19,20,26,29,42)$. A study claimed that participants with higher secondary and undergraduate education were at less risk of stress than the graduate (20). Contradictorily, respondents with secondary and undergraduate education were approximately 12.87-fold times and 1.12-fold times higher risk of anxiety symptoms than the graduates (20). Additionally, the secondary education cohort was at 2.30 times higher risk of suffering from depression than the graduates (20). Another study reported that participants with higher secondary education were more prone to develop depression and anxiety (26). Anxiety symptoms were less in having intermediate (11th -12th grade) education (about 0.5 times) and having bachelor education (approximately 0.64 times) than those with having higher education (above bachelor) (29).

f. Residence and family size

Living areas and the size of families were independently found as the risk factors for mental health suffering among individuals $(19,35,41,42)$. Living in an urban area and families with more than 5 members reported having significant psychological issues $(35,38,42)$. In contrast, another study reported that having children less than five in a family experienced more mental health outcomes (19).

g. Having elderly and children in the family

Having elderly and children in the family increased the risk of mental health problems $(19,20,36)$. For instance, student participants having children less than five years in the family had experienced higher levels of depression and stress (19). Similarly, other studies reported that elderly > 50-year old's in the participants' families who were at high risk of COVID-19 experienced higher anxiety and depression symptoms $(20,36)$.

\subsubsection{Behavior and Health-related Risk Factors}

\section{a. Smoking and alcohol use}

Smoking and alcohol use significantly influenced the risk of mental health problems such as stress, depression and anxiety a few studies $(25,30,35,39)$. Mamun et al. $(30)$ reported that $15.6 \%$ of the participants were depressed who were involved with smoking, whereas this rate was $3.3 \%$ for alcohol users. Besides, another study reported that those who were smoking more frequently significantly experience a high score for stress and depression (39).

b. Social media exposure

Propaganda, falsehood, conspiracy theories etc., related to the pandemic were increased, whereas social media was found to be one of the prime sources for collecting COVID-19 related information (24). Therefore, constantly using social media was a significant risk factor for mental health sufferings, which was also found in some studies $(24,27)$. For instance, $56.0 \%$ anxiety was found, with these participants reporting more than 4 hours of social media exposure, whereas it was $42.1 \%$ for $\leq 2$ hours (27). Similarly, a higher depression prevalence rate was observed in those physicians who reported it too hard to stay away from social media ( $58.3 \%$ vs. $47.8 \%)$ (24).

c. Physical exercise 
The physical exercise patterns had been changed after the inception of the COVID-19 pandemic, which was reported significantly associated with psychological burdens. For instance, participants who were not involved or inadequately involved with physical exercise generally experienced higher levels of depression and anxiety $(25,33,35)$.

\section{d. Physical health conditions}

Comorbidities were found to be a significant risk factor during the COVID-19 pandemic, leading to higher mental health problems. In contrast, the presence of several chronic diseases (i.e., hypertension, asthma, diabetes, heart diseases, chronic kidney disease, thyroid disorder) were also significantly influenced mental health issues $(8,22,30,36)$. Besides, due to more physical health problems, more severe psychological disorders were observed; for instance, it was reported among general people (30).

e. Psychological problems

A few studies reported other psychological problems associated with depression, anxiety, and stress. For instance, insomnia had been reported to have influenced the risk of mental health sufferings among university students and general people $(30,35)$. However, the physicians who reported sleeping less than 6 hours had significantly associated higher depression and anxiety symptoms (24).

\subsubsection{COVID-19 related Risk Factors}

\section{a. Insufficient protective resources}

The shortage of protective tools was a risk factor for psychological distresses during the COVID-19 pandemic. Few studies reported that the lack of personal protection equipment, inadequate or insufficient training, and other incentives related to protection influenced the risk of mental health problems among healthcare professionals $(22,24,25)$. Besides, Sakib et al. (36) found that only $15.9 \%$ of the healthcare professionals were satisfied with the quality of the PPE provided by the authorities, and the prevalence of depression was higher among HCPs who were dissatisfied with PPE (30.2\% vs. $18.0 \%)$. However, no studies that reported PPE-related issues in respect to mental health were conducted in other cohorts.

\section{b. COVID-19 related perceptions}

Peoples' perception of the COVID-19 pandemic significantly impacted their mental health. A study found that participants who had the lowest confidence in their current living place due to the COVID-19 pandemic and were highly concerned about their earning showed enormously significant associations with higher mental disorders (8). Whereas, in the case of perception of the current social life, satisfied respondents were less likely to have depression, anxiety and stress (8). In addition, perceptions related to (i) normal life disruption caused by the COVID-19 pandemic, (ii) its negative mental health effect of the pandemic, (iii) its effects on the healthcare system, leading people not be able to get enough medical care, (iv) for Bangladesh, the COVID-19 pandemic does/will not be a major problem, (v) its negative impact on education etc. had been observed being associated with mental health problems $(19,21)$.

\section{c. Fear of COVID-19 infection}

Most of the studies reported fear of COVID-19 infection influencing the risk of mental health problems, whereas fear of getting infected by the SARS-CoV-2 either themselves or their family members or relatives were the risk factors $(30-34,39,42)$. For instance, bankers reported that they were fearful of getting infected by the SARS-CoV-2 as their colleagues became infected, which later developed mental health problems (more specifically depression) among the other bankers (39).

\section{d. COVID-19 related symptoms}

People feeling or suffering from the COVID-19 related symptoms were at increased risk of mental disorders $(8,19)$. A study among the students found having more than one symptom had higher odds of stress and anxiety levels compared to those with one symptom (3.06 vs 1.60 , and 4.96 vs. 3.02, respectively; reference: no symptom]) (19). Correspondingly, another study observed that participants who had COVID-19 like symptoms, including fever, fatigue, and dry cough in the last 14 days, were significantly prone to suffering from depression, anxiety and stress (8).

e. Being suspected/infected with the COVID-19

Individuals who suspected or confirmed that they had close or indirect contact with the confirmed cases of COVID-19 were significantly suffered from higher levels of anxiety, depression and stress (8). Similarly, 3.17- and 3.99- times higher risk of anxiety and stress

Page $17 / 23$ 
suffering were observed to these participants reporting contact with individuals with confirmed cases of COVID-19 (19).

f. Being quarantined or isolated

Quarantine and isolation are the situations that generally increase the higher risk of mental health problems through developing trauma among the quarantined or isolated individuals. A study found that participants reporting to be quarantined 3.67-fold anxious than those who were not quarantined (19). Another study by Ripon et al. (23) reported that females generally developed a 4.7- and 4.8-times higher risk of PTSD and depression among home-quarantined people than male-quarantined people.

g. Healthcare facility and patient-related information

Physicians reporting inadequate resource to deal with the COVID-19 infected patients were at increased risk of depression and/or anxiety; that is, 2.28-, 5.30-, and 2.99-times higher risk were predicted for the mild, moderate and severe levels of depression and/or anxiety, respectively (22). Thus, healthcare professionals were being reported restlessness while examining the patients with flu-like symptoms, whereas a double depression rate was identified to these physicians compared to those who were not restless (36). Consequently, physicians who reported to be ready to deal with a COVID-19 positive patient had experienced higher levels of depression and anxiety (24).

\section{Discussion}

To the best of the author's knowledge, this is the first systematic review on common mental health problems during the COVID-19 pandemic in Bangladesh $(43,44)$, which has potential implications for mental health policymaking and practice. It is anticipated that the findings presented herein can be implemented for (i) developing cost-effective mental health strategies, (ii) prioritizing and integrating mental health in existing healthcare systems, (iii) improving access to information and mental health resources, (iv) addressing mental health disparities across different cohorts, (v) mobilizing social and community resources and organizations, (vi) strengthening mental health systems for the forthcoming public health emergencies etc. (45).

After investigating 24 recruited studies related to the COVID-19 pandemic, the overall range for the prevalence of depression, anxiety, and stress included $12.1-82.4 \%, 10.6-81.8 \%$ and $11.1-85.6 \%$, respectively, across diverse Bangladeshi cohorts. The higher rates of depression, anxiety, and stress was $82.4 \%, 81.8 \%$, and $85.6 \%$, respectively, in the university students (both anxiety and depression) and the general population (stress); whereas all of the lower rates were found among bankers (that is, $12.1 \%, 10.6 \%$ and $11.1 \%$, respectively). Most importantly, the prevalence rates of mental disorders vary within a specific group (general population, for example) due to the utilization of separate scales along with discrete cutoff values. Besides this, the sampling method and non-representativeness of the studies may also limit its reproducibility. In addition, there may have an effect of the COVID-19 exposure rate of the participants' area, personal and familiar history of the COVID-19 infection, financial conditions, and so forth lockdown related issues, which are not possibly identified from the included studies for concluding an overall assumption on mental disorders' prevalence rates across the cohorts. However, despite these limitations of the included studies, this review, for the first time, estimated the mental health problems' prevalence rates, which are anticipated to be worthy of consideration as a cautious situation rather than factual findings.

The COVID-19 pandemic has dramatically interchanged the consistent activities of individuals to prevent and control the outbreak. Under this circumstance, existing healthcare facilities in the country transformed into increased demands beyond its existence as competent. During the normal period, effective and efficient health care services were allegedly absent in Bangladesh (12,46). Along with this, due to the shortage of adequate preventive equipment, frontline healthcare professionals frequently feel unsafe and get scared to be infected as they have to directly take care of the COVID-19 infected patients (24). Besides, many frontline healthcare professionals lost their lives worldwide being infected with the SARS-CoV-2 in their workplaces (47). Later on, this situation also upsurges psychological weight, including suicidal occurrences among the healthcare providers (48). Consistent with the scenario, the physicians who provided services in the Dhaka division were more susceptible to being anxious than other divisional physicians [41], which may be due to being identified in most cases and deaths in the Dhaka division.

Applying some of the mandatory restrictions, including social distancing at the earlier period of the pandemic inception, people started grabbing and worrying about their lives and personal safety, fear of getting infected, lost a beloved one, absence of appropriate treatment etc., which influenced the risk of depression, anxiety and stress $(17,45)$. Thus, participants belonging to general people, students etc. cohorts had to experience mental health problems. Besides this, daily wage-earners were at higher risk of adverse psychological effects as they could not meet their family's livelihood due to the countrywide lockdown and losing their jobs (14). Despite the wage-earners being a risky group, only a study was conducted within this cohort. Similarly, a study was conducted for other vulnerable cohorts, e.g.,

Page 18/23 
COVID-19 infected people, COVID-19 suspected people and bankers. Through the RT-PCR test, the individuals diagnosed with the COVID19 generally got scared about the risk of mortality, which affects their adverse psychological consequences. For similar reasons, the quarantined people were found experiencing detraction of psychological wellbeing (23). In addition, bankers were also at higher risk of being affected psychologically as they had to deal with the clients during the pandemic since the banks were not completely shut down. Therefore, their colleague's news of being infected got scary, although the lower prevalence rates of anxiety, depression, and stress were reported to this cohort (39). Nevertheless, only a study was directed upon this mentally susceptible group (39), which may result in vague pictures concluding bankers compared to other cohorts are not at risk of higher mental health problems as reported in the only study.

This review also revealed the associated risk factors that influenced the incidence of psychological consequences during the COVID-19 pandemic situation in Bangladesh. Based on the present review, socio-demographic factors including gender (mostly being female), younger people, living in an urban area, family size (more than 5 members), being unemployed, marital and educational status, having children and elderly in a family influenced the risk of being mentally affected $(20,24-26,29,35)$. Furthermore, it was observed that people who had existing physical health-related comorbidities developed higher psychological problems $(22,30)$. Besides this, being physically inactive and constantly using social media and watching news reported increasing the risk of mental instabilities $(24,27)$. In addition, the COVID-19 related perceptions, experiencing the COVID-19 related symptoms, being suspected or infected with the SARS-CoV-2, being contacted with the COVID-19 confirmed cases, being quarantined or isolated etc., were reported as the psychological suffering risk factors. Finally, the fear of being infected with the virus due to the scarcity of appropriate preventive equipment and inadequate resources in healthcare settings to deal with the COVID-19 infected patients were also reported as a risk factor of those psychological consequences (24). In the end, it can be said that any of the COVID-19 related adverse outcomes increased the risk of higher mental health problems.

There are several limitations of the present review to be mentioned. First of all, potential studies that could have met this review inclusion criterion were not included beyond the strategies, which may limit the review by selection bias; although databases like Google Scholar, ResearchGate, even Google search engine were used for screening additional articles, and the research team tried their best to find out all of the eligible articles. Preprint articles were included in this review in view of disseminating the available relevant information; however, these articles being non-peer-reviewed can be one of the limitations of study inclusion. In addition, no quality assessment was done to this review. Besides this, the overview of mental disorders' prevalence rates reported in this review can be limited; because many of these articles did not follow similar strategies for cutoff point and inter-studies-related limitations as aforementioned. Despite these limitations, the present review provides an overall estimation of the mental health problems (e.g., depression, anxiety, stress) in the context of Bangladesh, which may have potential influences on adopting preventive mental health strategies including knowledge-exchange and training people through employing online-based intervention programs along with providing psychological cares, implementing cohortbased intervention program, arranging series webinar with a special focus on mental health, telecasting positivity about COVID-19 on media channel by taking as caution rather than factual findings. In addition, this review's limitations are highly suggested to be addressed in future reviews adopting a more rigorous approach; for instance, for estimating pooled prevalence rates, meta-analytic reviews should be performed.

\section{Conclusions}

The COVID-19 pandemic has become a global public health threat due to its devastating effects on peoples' life throughout the world. To facilitate mental health policymaking and practice, there is no beyond integrated information. Therefore, the mental disorders during the COVID-19 pandemic in Bangladesh are reviewed herein for the first time, which is the major strength to be noted. From this review, the provided baseline information related to prevalence and risk factors of depression, anxiety, and stress across different cohorts can be utilized in the policy levels to adopt appropriate preventive measures and practice to alleviate psychological consequences and physical health problems.

\section{Declarations}

Declarations - Ethics approval

Not applicable to this study.

Declarations - Consent for publication

Not applicable to this study. 
Declarations - Availability of data and materials

Not applicable to this study.

Declarations - Competing interests

The authors of the research work do not have any conflict of interest.

Declarations - Funding

The present study did not get any financial support.

Declarations - Authors' contributions

All the authors contributed equally to this work.

Declarations - Acknowledgments

The authors like to acknowledge that their affiliation with the CHINTA Research Bangladesh, was formerly known as the Undergraduate Research Organization.

\section{References}

1. UNDP. COVID-19 Pandemic Response. (2020) [cited 7 Apr 2021]. Available: https://www.undp.org/content/undp/en/home/coronavirus.html.

2. Puspitasari IM, Yusuf L, Sinuraya RK, Abdulah R, Koyama H. Knowledge, Attitude, and Practice During the COVID-19 Pandemic: A Review. J Multidiscip Healthc. 2020;13:727-33. doi:10.2147/JMDH.S265527.

3. BBC NEWS. Coronavirus confirmed as pandemic by World Health Organization. (2020) [cited 23 Mar 2021]. Available: https://www.bbc.com/news/world-51839944.

4. Chen N, Zhou M, Dong X, Qu J, Gong F, Han Y, Qiu Y, Wang J, Liu Y, Wei Y, et al. Epidemiological and clinical characteristics of 99 cases of 2019 novel coronavirus pneumonia in Wuhan, China: a descriptive study. Lancet. 2020;395:507-13. doi:10.1016/S01406736(20)30211-7.

5. CDC. Symptoms of Coronavirus. (2020) [cited 23 Mar 2021]. Available: https://www.cdc.gov/coronavirus/2019-ncov/symptomstesting/symptoms.html.

6. Rajkumar RP. COVID-19 and mental health: A review of the existing literature. Asian J Psychiatr. 2020;52:102066. doi:10.1016/j.ajp.2020.102066.

7. Bao Y, Sun Y, Meng S, Shi J, Lu L. 2019-nCoV epidemic: address mental health care to empower society. Lancet. 2020;395:e37-8.

8. Rahman MM, Khan SJ, Sakib MS, Chakma S, Procheta NF, Mamun Z, Al, Arony A, Rahman F, Rahman MM. Assessing the psychological condition among general people of Bangladesh during COVID-19 pandemic. J Hum Behav Soc Environ (2020)1-15. doi:10.1080/10911359.2020.1848688.

9. Gualano MR, Lo Moro G, Voglino G, Bert F, Siliquini R. Effects of Covid-19 lockdown on mental health and sleep disturbances in Italy. Int J Environ Res Public Health. 2020;17:4779. doi:10.3390/ijerph17134779.

10. Dsouza DD, Quadros S, Hyderabadwala ZJ, Mamun MA. Aggregated COVID-19 suicide incidences in India: Fear of COVID-19 infection is the prominent causative factor. Psychiatry Res. 2020;290:e113145. doi:10.1016/j.psychres.2020.113145.

11. Hawryluck L, Gold WL, Robinson S, Pogorski S, Galea S, Styra R. SARS control and psychological effects of quarantine, Toronto, Canada. Emerg Infect Dis. 2004;10:1206-12. doi:10.3201/eid1007.030703.

12. Anwar S, Nasrullah M, Hosen MJ. COVID-19 and Bangladesh: Challenges and how to address them. Front public Heal (2020) 8: doi:10.3389/fpubh.2020.00154.

13. Bhuiyan AKMI, Sakib N, Pakpour AH, Griffiths MD, Mamun MA. COVID-19-related suicides in Bangladesh due to lockdown and economic factors: Case study evidence from media reports. Int J Ment Health Addict (2020)epub ahead of print. doi:10.1007/s11469-020-00307-y.

14. Sultana MS, Khan AH, Hossain S, Islam T, Hasan MT, Ahmed HU, Khan JAM. The association between financial hardship and mental health difficulties among adult wage earners during the COVID-19 pandemic in Bangladesh: Findings from a cross-sectional

Page 20/23 
analysis. PsyArXiv. 2020. doi:10.31234/osf.io/q3ehv.

15. Sundarasen S, Chinna K, Kamaludin K, Nurunnabi M, Baloch GM, Khoshaim HB, Hossain SFA, Sukayt A. Psychological impact of Covid-19 and lockdown among university students in Malaysia: Implications and policy recommendations. Int J Environ Res Public Health. 2020;17:6206. doi:10.3390/ijerph17176206.

16. World Health Organization. COVID-19 disrupting mental health services in most countries. WHO survey. (2020) [cited 23 Mar 2021]. Available: https://www.who.int/news/item/05-10-2020-covid-19-disrupting-mental-health-services-in-most-countries-who-survey.

17. Hossain MM, Rahman M, Trisha NF, Tasnim S, Nuzhath T, Hasan NT, Clark H, Das A, McKyer ELJ, Ahmed HU. Prevalence of anxiety and depression in South Asia during COVID-19: A systematic review and meta-analysis. PsyArXiv Prepr (2020).

18. Moher D, Liberati A, Tetzlaff J, Altman DG. Preferred reporting items for systematic reviews and meta-analyses: the PRISMA statement. Int J Surg. 2010;8:336-41.

19. Sayeed A, Kundu S, Al Banna MH, Hasan MT, Begum MR, Khan MSI. Mental health outcomes during the COVID-19 and perceptions towards the pandemic: Findings from a cross sectional study among Bangladeshi students. Child Youth Serv Rev. 2020;119:105658. doi:10.1016/j.childyouth.2020.105658.

20. Banna M, Al, Sayeed A, Kundu S, Christopher E, Al Banna MH, Sayeed A, Kundu S, Christopher E, Hasan MT, Begum MR, et al. The impact of the COVID-19 pandemic on the mental health of the adult population in Bangladesh: A nationwide cross-sectional study. PsyArXiv (2020)0-19. doi:10.31234/osf.io/chw5d.

21. Islam SMDU, Bodrud-Doza M, Khan RM, Haque MA, Mamun MA. Exploring COVID-19 stress and its factors in Bangladesh: A perception-based study. Heliyon. 2020;6:e04399. doi:10.1016/j.heliyon.2020.e04399.

22. Barua L, Zaman MS, Omi FR, Faruque M. Psychological burden of the COVID-19 pandemic and its associated factors among frontline doctors of Bangladesh: a cross-sectional study. F1000Research (2020) 9:1304.

23. Ripon RK, El-Sabban F, Sikder T, Hossain S, Mim SS, Ahmed HU, Mehta N. Psychological and nutritional effects on a COVID-19quarantined population in Bangladesh. J Hum Behav Soc Environ (2020)1-12. doi:10.1080/10911359.2020.1822252.

24. Hasan MT, Hossain S, Safa F, Anjum A, Khan AH, Koly KN, Alam SF, Rafi MA, Podder V, Trisa TI. Prevalence of anxiety and depressive symptoms among physicians during the COVID-19 pandemic in Bangladesh: a cross-sectional study. medRxiv. 2020. doi:10.1101/2020.12.08.20245829.

25. Tasnim R, Sujan MSH, Islam MS, Ritu AH, Siddique MA, Bin, Toma TY, Nowshin R, Hasan A, Hossain S, Nahar S. Prevalence and correlates of anxiety and depression in frontline healthcare workers treating people with COVID-19 in Bangladesh. PsyArXiv (2020) doi:10.31234/osf.io/3qg9p.

26. Ahmed O, Ahmed MZ, Alim SMAHM, Khan MDAU, Jobe MC. COVID-19 outbreak in Bangladesh and associated psychological problems: An online survey. Death Stud (2020)1-10. doi:10.1080/07481187.2020.1818884.

27. Hossain MT, Ahammed B, Chanda SK, Jahan N, Ela MZ, Islam MN. Social and electronic media exposure and generalized anxiety disorder among people during COVID-19 outbreak in Bangladesh: A preliminary observation. PLoS One. 2020;15:e0238974.

28. Islam MA, Barna SD, Raihan H, Khan MNA, Hossain MT. Depression and anxiety among university students during the COVID-19 pandemic in Bangladesh: A web-based cross-sectional survey. PLoS One. 2020;15:e0238162.

29. Islam MS, Ferdous MZ, Potenza MN. Panic and generalized anxiety during the COVID-19 pandemic among Bangladeshi people: An online pilot survey early in the outbreak. J Affect Disord. 2020;276:30-7. doi:10.1016/j.jad.2020.06.049.

30. Mamun MA, Sakib N, Gozal D, Bhuiyan AKMI, Hossain S, Bodrud-Doza M, Al Mamun F, Hosen I, Safiq MB, Abdullah AH. The COVID19 pandemic and serious psychological consequences in Bangladesh: a population-based nationwide study. J Affect Disord. 2020;279:462-72. doi:10.1016/j.jad.2020.10.036.

31. Al Zubayer A, Rahman ME, Islam MB, Babu SZD, Rahman QM, Bhuiyan MRAM, Khan MKA, Chowdhury MAU, Hossain L, Habib R. Bin. Psychological states of Bangladeshi people four months after the COVID-19 pandemic: an online survey. Heliyon. 2020;6:e05057. doi:10.1016/j.heliyon.2020.e05057.

32. Safa F, Anjum A, Hossain S, Trisa TI, Alam SF, Rafi MA, Podder V, Koly KN, Azad DT, Ahmad WU. Immediate psychological responses during the initial period of the COVID-19 pandemic among Bangladeshi medical students. Child Youth Serv Rev. 2021;122:105912. doi:10.1016/j.childyouth.2020.105912.

33. Khan AHH, Sultana MSS, Hossain S, Hasan MT, Ahmed HU, Sikder MTT. The impact of COVID-19 pandemic on mental health \& wellbeing among home-quarantined Bangladeshi students: A cross-sectional pilot study. J Affect Disord. 2020;277:121-8.

34. Faisal RA, Jobe MC, Ahmed O, Sharker T. Mental Health Status, Anxiety, and Depression Levels of Bangladeshi University Students During the COVID-19 Pandemic. Int J Ment Health Addict (2021)1-16. doi:10.1007/s11469-020-00458-y.

Page 21/23 
35. Islam MS, Sujan MSH, Tasnim R, Sikder MT, Potenza MN, Van Os J. Psychological responses during the COVID-19 outbreak among university students in Bangladesh. PLoS One. 2020;15:e0245083. doi:10.1371/journal.pone.0245083.

36. Sakib N, Akter T, Zohra F, Bhuiyan AKMI, Mamun MA, Griffiths MD. Fear of COVID-19 and Depression: A Comparative Study Among the General Population and Healthcare Professionals During COVID-19 Pandemic Crisis in Bangladesh. Int J Ment Health Addict. 2021. doi:10.1007/s11469-020-00477-9.

37. Mina FB, Billah M, Karmakar S, Das S, Rahman MS, Hasan MF, Acharjee UK. An online observational study assessing clinical characteristics and impacts of the COVID-19 pandemic on mental health: A perspective study from Bangladesh. J Public Health (Bangkok) (2021)1-9. doi:10.1007/s10389-020-01445-2.

38. Dhar BK, Ayittey FK, Sarkar SM. Impact of COVID-19 on Psychology among the University Students. Glob Challenges. 2020;4:2000038. doi:10.1002/gch2.202000038.

39. Yasmin S, Alam MK, Ali F, Bin, Banik R, Salma N. Psychological Impact of COVID-19 Among People from the Banking Sector in Bangladesh: a Cross-Sectional Study. Int J Ment Health Addict (2021)1-15. doi:10.1007/s11469-020-00456-0.

40. Khatun MF, Parvin MF, Rashid MM, Alam MS, Talukder A, Razu SR, Ward PR, Ali M. Mental Health of Physicians During COVID-19 Outbreak in Bangladesh: A Web-Based Cross-Sectional Survey. Front public Heal (2021) 9: doi:10.3389/fpubh.2021.592058.

41. Dhar BK, Ayittey FK, Sarkar SM. Impact of COVID-19 on Psychology among the University Students. Glob Challenges. 2020;4:2000038. doi:10.1002/gch2.202000038.

42. Yeasmin S, Banik R, Hossain S, Hossain MN, Mahumud R, Salma N, Hossain MM. Impact of COVID-19 pandemic on the mental health of children in Bangladesh: A cross-sectional study. Child Youth Serv Rev. 2020;117:105277. doi:10.1016/j.childyouth.2020.105277.

43. Ahmed F, Sifat RI. Strategic assessment of mental health and suicide amid COVID-19 pandemic in Bangladesh. Int J Health Plann Manage n/a: doi:https://doi.org/10.1002/hpm.3121.

44. Sifat RI. COVID-19 pandemic: Mental stress, depression, anxiety among the university students in Bangladesh. Int J Soc Psychiatry (2020)20764020965995. doi:10.1177/0020764020965995.

45. Hossain MM, Tasnim S, Sultana A, Faizah F, Mazumder H, Zou L, McKyer ELJ, Ahmed HU, Ma P. Epidemiology of mental health problems in COVID-19: a review. F1000Research (2020) 9: doi:10.12688/f1000research.24457.1.

46. Al-Zaman MS. Healthcare Crisis in Bangladesh during the COVID-19 Pandemic. Am J Trop Med Hyg. 2020;103:1357-9.

47. Bandyopadhyay S, Baticulon RE, Kadhum M, Alser M, Ojuka DK, Badereddin Y, Kamath A, Parepalli SA, Brown G, Iharchane S. Infection and mortality of healthcare workers worldwide from COVID-19: a systematic review. BMJ Glob Heal (2020) 5:e003097. doi:10.1136/ bmjgh-2020-003097.

48. Jahan I, Ullah I, Griffiths MD, Mamun MA. COVID-19 suicide and its causative factors among the healthcare professionals: Case study evidence from press reports. Perspect Psychiatr Care(2021) n/a: doi:10.1111/ppc.12739.

\section{Figures}




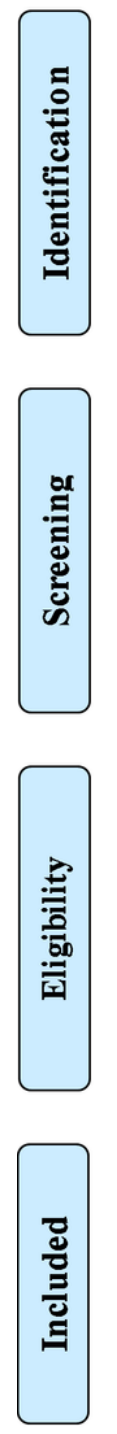

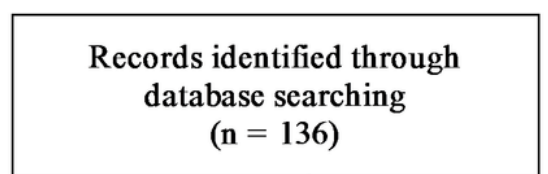

$(\mathrm{n}=136)$
Additional records identified through other sources

$(\mathrm{n}=39)$

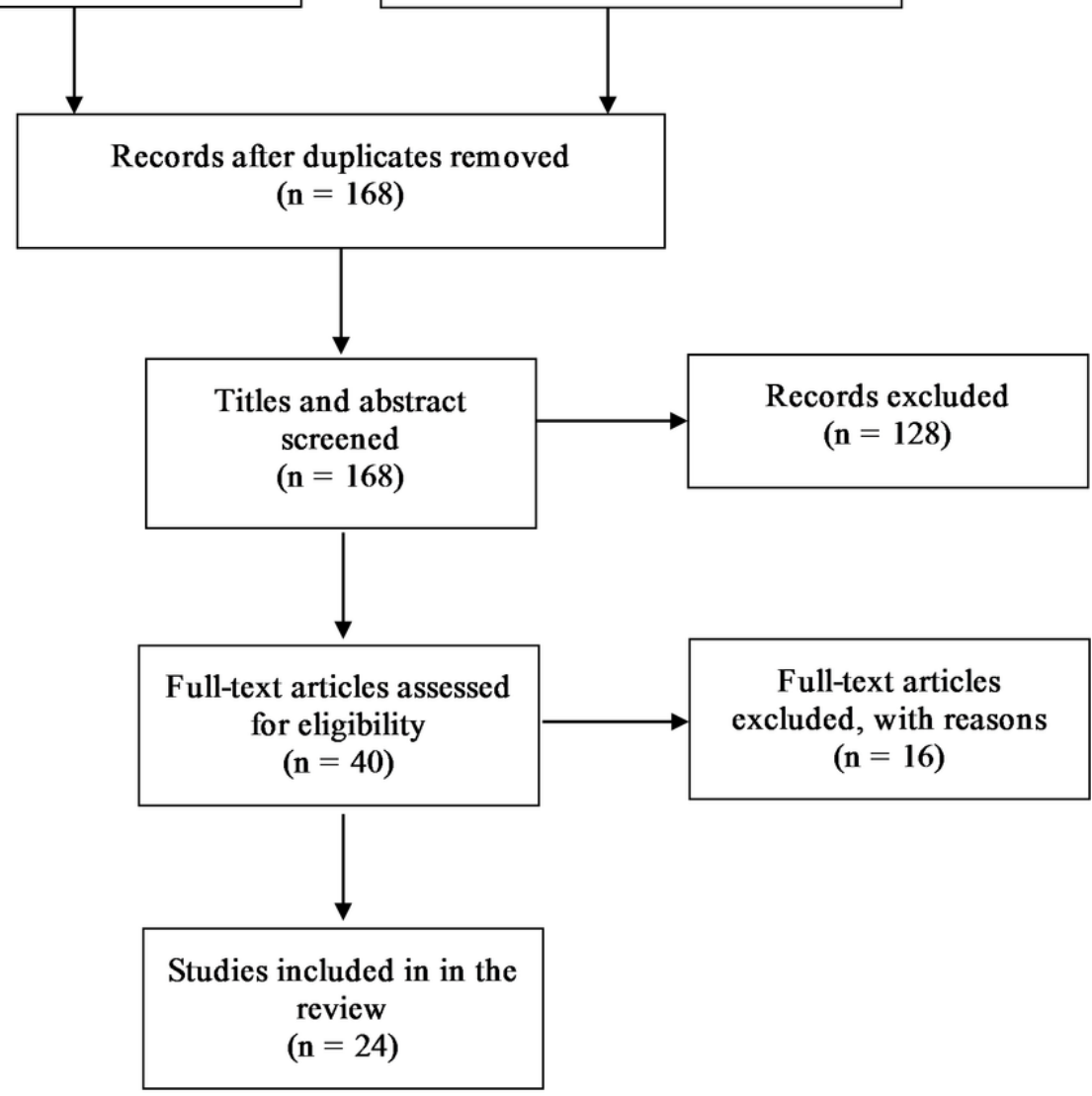

\section{Figure 1}

PRISMA Flowchart of the Present Review 